\title{
STUDENTS' EXPERIENCE OF STRESS WITH DIFFERENT FRAMEWORK CONDITIONS AND DIFFERENT ORIGINS
}

\author{
Irina Böckelmann ${ }^{1}$,Håvard R. Karlsen ${ }^{2}$, Sabine Darius ${ }^{1}$, Beatrice Thielmann ${ }^{2}$ \\ ${ }^{1}$ Institute of Occupational Medicine, Medical Faculty, \\ Otto-von-Guericke-University, Magdeburg, Germany \\ ${ }^{2}$ Department of Psychology, Norwegian University \\ of Science and Technology, Trondheim, Norway
}

https://doi.org/10.35339/ic.8.2.74-86

\begin{abstract}
Background: Students are exposed to numerous stress factors. The large number of demands and high strain can lead to a higher drop-out rate. For this reason, the aim of the study is to examine and comparing the experience of stress among German and international students during their studies under adaptation to generally stressful conditions. The international students performed a complete study program in Germany. Methods: Data from 194 students $(41.8 \%$ women, $58.2 \%$ men) were evaluated. The average age of the participants was 23.0 3.44 years. Our sample consisted of 80 international students. The questionnaire on strains during the study (and at the workplace) was used. We registered the frequency and the intensity of the stress factors in everyday study life. We factor analyzed the 34 stress items, which gave five stress factors. We then looked at the differences between German and international students on these factors, while adjusting for generally stressful conditions. Results: The most important stressors were unfavorable working hours, incompatibility of tasks, climate, excessive demands, high responsibility, lack of information, social isolation, emotional strain and financial problems. The stressors differed in 17 out of 34 stressors in the groups of students of different origin. The international students experienced more physical and psychosocial strain and they experienced resources less often than German students did, but if they felt them, it was more intense. Conclusions: Strains during studies and their individual demands vary. German and international students have different perceptions of stress during study programs. There is a need for health promotion and prevention programs, which should be integrated during study.
\end{abstract}

Keywords: Students, stress factors, international students, strain, demand, factor analysis.

\section{Introduction}

\section{Background}

The initiation of the Bologna reforms resulted in extensive changes in the demand and resource profiles of students in Europe, which made a systematic health reporting necessary [1]. The number of students has grown significantly in recent years [2]. Current studies showed that one out of every two students was regularly under stress [1]. When asked about stress-related factors, $75 \%$ of the students associated those with time pressure, $64 \%$ with performance pressure and almost $40 \%$ with fear of the future

Corresponding Author:

Beatrice Thielmann, Dr. med., Institute

of Occupational Medicine, Medical Faculty,

Otto-von-Guericke-University, Magdeburg, Germany.

E-mail: beatrice.thielmann@med.ovgu.de and work overload. Along with a clear feeling of exhaustion in almost $50 \%$ of cases, exposure to multiple sources of stress is of great importance $[3,4]$. In addition to the stressors of environmental conditions, such as noise, seating arrangements in lecture halls and their lack of clarity, numerous psychological stressors were named, such as the increasing time pressure and need to rush [5]. Common sources of stress among students are their financial situation, part-time work, relationships, caring for children and own health [4, $6,7]$. Stressors may have an adverse effect on the chance of students to complete their study program successfully.

\section{Effects of study-related stress on the course of study}

It has been shown that the volume of stressful life experiences is exponentially associated with 
the average perceived impairment of academic performance [8]. There are noticeable differences between bachelor and master students [1]. Bachelor students face the added pressure of getting into a good master's program that the master students do not have to worry about. Pressure by forthcoming examinations, performance demands as well as the financial situation seems to be significantly reduced for master students, according to previous studies. Higher numbers and intensity of demands can lead to an elevated drop-out rate [9]. Approximately one quarter of these dropouts is due to failure to achieve performance requirements and one in two is due to excessive study requirements [10]. According to the German Academic Exchange Service in 2012, $28 \%$ of German students dropped out of their study, compared to $46 \%$ of international students in Germany.

\section{The mental health of students}

Psychosocial problems, exhaustion, psychosomatic complaints or fears resulting in sleep disorders, nervousness, concentration problems or mood swings are prevalent among German students [11]. Students showed increased alcohol consumption [12]. While male students are more likely to consume alcohol or stimulants [7, 13, 14], female students show other spontaneous reactions to the stressors, for example, crying, eating or being restless [15]. The dysfunctional use of social media is also widespread and results in pronounced concentration problems and mental disorders due to a lack of functional solutions [16]. In addition to the study and the workplace, the predominant areas of life are health, family situation and partnership, including social contacts. Studies show that about $27 \%$ of students are suffering from psychological impairments. The rate of depressive disorders in the student population is $18 \%$, which is significantly higher than the German population average of 5\% [17]. Another study showed that the risk of burnout prevalence doubled from the third to sixth year of study of medicine [18].

2. Purpose, subjects and methods:

2.1. The purpose of this international study is to measure and compare the common stressors among German and international students and examine these stressors in relation to the student's background and general conditions. We hypothesized a differing perception of stress. The following questions were examined:

- Are there differences in the perception of the frequency and the intensity of study- or workrelated stressors among German and international students?
- Do these differences remain unchanged after controlling for other generally stressful conditions?

\subsection{Subjects \& Methods}

The international students that came to Germany to perform a full study program at a university were recruited. The subjective stress-strain concept was applied as a theoretical method [19].

In the survey 194 students (41.8\% women $(\mathrm{n}=81)$ and $58.2 \%$ men $(\mathrm{n}=113))$ was participated. The students came from diverse faculties of the two universities. The surveys were conducted in the period 2010-2013 and 2018. The average age of the participants was $23.0 \pm 3.44$ years (range: 18-34 years). The sample comprises 80 international participants among 36 countries (e. g. Cameroon $(n=9)$, Syria $(n=7)$, Palestine $(\mathrm{n}=4)$, Russia $(\mathrm{n}=4)$, Bulgaria $(\mathrm{n}=4)$, Israel $(\mathrm{n}=4)$, Peru ( $\mathrm{n}=3)$, Argentina $(\mathrm{n}=3)$, Georgia $(\mathrm{n}=3)$, Iran $(\mathrm{n}=2)$, Yemen $(\mathrm{n}=2)$, and other countries all over the world with one or two students. They aged between 20 and 30 years $(24.7 \pm 2.40$ years). The average age of German students was slightly lower $(21.7 \pm 3.53$ years $)$ compared to the international students. The gender ratio within the groups of students with and without German origin was relatively similar $(\mathrm{p}=0.442)$ : 45 German women $(39.5 \%)$ and 69 German men $(60.5 \%)$ vs. 36 international women (45.0\%) and 44 international men $(55.0 \%)$.

The call for voluntary participation to the study was made through flyers with information and contact details. As well as the questionnaires laid out in paper format, which were available to all present at the first semester event in the lecture hall. The students filled out independently without any time limit. In the case of language barriers of international students (all questions were in German), an interpreter assisted in understanding and answering the respective questions.

Questionnaire on strains during the study (and at the workplace)

This questionnaire collects socio-demographic data of the participants as well as information on the study program, the time in hours per week required for the study, the financing of the study program, possible employment and the individual protective factors of the students [20]. In addition, the strain and the resulting demands during the study and the possible secondary employment were queried. The first step is an evaluation of the existence of the stressor, whether the respective statement (stress) occurs in everyday study life (never, rarely, sometimes, often). In the second step, the strength of the stressor is evaluated on 
a four-level scale (as not at all, a little, quite strong or very strong). Thus, this questionnaire reflects typical stressors in the categories of psychological and physical stress, stress in connection with the financing of the study program, time stress during the study program and also family stress.

The students were asked about the frequency and the intensity of the following stress factors: time pressure, noise exposure, dirt pollution, physically heavy work, forced posture, lifting/ carrying, unfavorable working hours, incompatibility of tasks, dangerous substances pollution, remain seated for long periods, long daily journey, risk for infection, accident risks exposure, climate, monotony, work overload, work underload, high accountability, little room for maneuver, no organization possibilities, lack of information, sexual harassment, conflicts with the faculty, conflicts with colleagues, conflicts with customers/patients, social isolation, fear of unemployment, emotional stress, problems in dealing with personal computers, bullying, financial difficulties, work-family conflict because of study/work, enjoy studying, collegial and open atmosphere in the environment, support from family/partner in professional matters.

At last, the following four factors were considered generally stressful conditions: working alongside studying, caring for children, nursing relatives and travel distance to place of work or study of more than 120 minutes. Of the total sample, these factors were cited as the main stressors. Therefore, they were included in the factor analysis. Based on the results of the potentially general stressful conditions, the students were divided into subgroups without $(\mathrm{n}=108(55.7 \%))$ and with ?1 stressful condition; $\mathrm{n}=86(44.3 \%)$.

Statistical methods

All analyses were performed in Stata v.16. We performed a factor analysis on the items, with principal (axis) factors extraction method (PF), because the items had a high degree of uniqueness. We tested the appropriateness of using factor analysis by running Bartlett's test of sphericity and calculating the Kaiser-Meyer-Olkin Measure of Sampling Adequacy (KMO). We did this procedure separately for the items measuring stress through frequency and the ones measuring stress through intensity. The items that loaded highly and uniquely on each factor were then combined into a composite factor score. These factor scores were then used in Mann-Whitney$\mathrm{U}$ tests to compare the scores of German and international students. We also calculated regression models with the factor scores as the dependent variable and origin (German vs. international) as the independent variable, while adjusting for the effect of generally stressful conditions. Here we used robust standard errors because most of the regression models showed heteroskedasticity and non-normal residuals.

3. Results \& Discussion

Results

For 108 of 194 students (55.7\%), there were no potentially stressful general conditions that could affect their study: 57 (50.0\%) German students and $51(63.75 \%)$ international students $(\mathrm{p}=0.058)$. For 86 participants $(44.3 \%)$ potentially stressful general conditions were found $(38.7 \%$ with one, $5.2 \%$ with two and $0.5 \%$ with at least three). Of the 57 students of German origin with potentially stressful general conditions, 46 had only one, 10 had two and one German student had more than two potentially stressful general conditions. All 29 international students stated that they had only one potentially stressful general condition. There was no significant difference in the distribution of participants with generally stressful conditions among German and international students $(p=0.058)$. The average age of students without stressful conditions was $22.5 \pm$ 3.13 years, similar to the average age of those with stressful conditions $23.5 \pm 3.72$ years.

The average travel time to the university of $8.2 \pm 15.67 \mathrm{~km}$ was $21.6 \pm 18.19$ minutes. The participants reported that they spent on average 19.2 \pm 9.39 hours per week on lectures, seminars and traineeships. They spent an average of $15.6 \pm 10.89$ hours per week on the preparation and follow-up. Only four German students (2.1\% of the total sample) had children. Twenty-seven $(23.9 \%)$ German students had their studies financed $100 \%$ by their parents, compared to 39 $(48.8 \%)$ of the international students. Only two German (1.8\%) and six (7.5\%) international students financed their studies completely though their own income. Most students received some sort of funding through some combination of parents, own income and/or scholarships or similar arrangements.

The frequency of stressors during the studies and/or possible work during the studies were surveyed. The most frequently reported stressors in the overall sample were time pressure (which $34 \%$ reported feeling often) and long periods of sitting $(39.2 \%)$. These were followed by unfavourable working hours, incompatibility of tasks, climate, excessive demands, high respon-sibility, lack of information, social isolation, emotional strain, and financial problems. 
German students more frequently reported enjoying the study and work as well as the collegial and open atmosphere in the environment. $35.7 \%$ of German students answered the question "enjoyment of studies and work" with "sometimes" and $58.9 \%$ with "often" (vs. $48.8 \%$ and $28.7 \%$ in the other group). They also felt more support (considering the answers "sometimes" and "often" together) for professional interests from their immediate circle $(90.2 \%$ vs. $80.0 \%)$.

After this analysis of individual stressors, a factor analysis was undertaken. The KMO was 0.758 , which was above the acceptable threshold of 0.6, and indicated that the sample was big enough to show patterns that could yield factors. Bartlett's test of sphericity was significant at $p<.001$, which indicated that the correlation matrix of all variables in the factor analysis was significantly different from an identity matrix. Taking together, this indicated that factor analysis could be performed.
A factor analysis with principal (axis) factors extraction method (PF) was performed on the 34 variables measuring the frequency of stressors. Parallel analysis indicated that five factors should be extracted: factor A physical strain, factor B psycho-social strain, factor C conflicts, factor D organizational strain, and factor $\mathrm{E}$ resources. Together they explained $83 \%$ of the variance in the data. A promax rotation gave $22 \mathrm{va}-$ riables with factor loadings higher than 0.4 , without cross-loadings. The factors and their related items are presented in Table 1a. All factors had Cronbach's $\alpha$ above .70, apart from Factor $\mathrm{D}$ with $\alpha=.40$. Table $1 b$ shows the frequency of stressors in comparison of German and international students that had particular influence on the factor analysis. Significantly more Germans than international students reported sometimes or often stressors such as physically heavy work, lifting/carrying, remain seated for long periods,

Factor analysis of items measuring frequency of stress factors among students

\begin{tabular}{|c|c|c|c|c|c|}
\hline Variable & Factor A & Factor B & Factor C & Factor D & Factor $\mathrm{E}$ \\
\hline Time pressure & 0,05 & 0,52 & 0,06 & 0,00 & 0,16 \\
\hline Noise exposure & 0,28 & 0,01 & 0,07 & 0,26 & 0,03 \\
\hline Dirt pollution & 0,82 & $-0,08$ & 0,00 & 0,01 & $-0,09$ \\
\hline Physically heavy work & 0,85 & 0,05 & $-0,02$ & $-0,02$ & $-0,08$ \\
\hline Forced posture & 0,31 & 0,25 & $-0,10$ & 0,27 & $-0,10$ \\
\hline Lifting/carrying & 0,88 & 0,00 & 0,04 & $-0,22$ & 0,03 \\
\hline Unfavourable working hours & 0,17 & 0,57 & $-0,19$ & 0,13 & 0,06 \\
\hline Incompatibility of tasks & 0,02 & 0,72 & $-0,07$ & 0,15 & 0,12 \\
\hline Dangerous substances pollution & 0,54 & 0,01 & 0,03 & 0,13 & $-0,11$ \\
\hline Sitting still for long periods & $-0,12$ & $-0,06$ & 0,02 & 0,62 & 0,22 \\
\hline Long daily journey & 0,05 & 0,19 & 0,07 & 0,29 & 0,11 \\
\hline Risk for infection & 0,20 & 0,15 & $-0,06$ & 0,33 & 0,15 \\
\hline Accident risks exposure & 0,57 & $-0,11$ & 0,03 & 0,20 & 0,02 \\
\hline Climate (coldness, hotness, draft, etc.) & 0,13 & 0,41 & $-0,11$ & 0,02 & $-0,13$ \\
\hline Monotony & $-0,03$ & 0,17 & $-0,10$ & 0,52 & $-0,07$ \\
\hline Work overload & $-0,16$ & 0,58 & 0,01 & 0,13 & 0,02 \\
\hline Work underload & 0,04 & $-0,25$ & 0,24 & 0,38 & 0,13 \\
\hline High accountability & 0,27 & 0,37 & 0,15 & $-0,11$ & 0,05 \\
\hline Little room for maneuver, no organization possibilities & $-0,23$ & 0,31 & 0,31 & 0,20 & $-0,11$ \\
\hline Lack of information & $-0,09$ & 0,17 & 0,17 & 0,41 & $-0,24$ \\
\hline Sexual harassment & $-0,02$ & $-0,15$ & 0,36 & 0,15 & $-0,02$ \\
\hline Conflicts with faculty & $-0,02$ & 0,05 & 0,57 & 0,12 & 0,08 \\
\hline Conflicts with colleagues & 0,04 & $-0,09$ & 0,74 & $-0,03$ & 0,07 \\
\hline Conflicts with customers/patients & 0,27 & 0,04 & 0,45 & $-0,29$ & 0,17 \\
\hline Social isolation & $-0,28$ & 0,43 & 0,00 & $-0,19$ & $-0,31$ \\
\hline Fear of unemployment & $-0,04$ & 0,13 & 0,35 & 0,00 & $-0,21$ \\
\hline Emotional stress & $-0,06$ & 0,59 & 0,02 & $-0,09$ & $-0,19$ \\
\hline Problems in dealing with personal computers & $-0,02$ & 0,07 & 0,37 & 0,15 & $-0,08$ \\
\hline Bullying & 0,01 & 0,20 & 0,37 & $-0,14$ & $-0,21$ \\
\hline Financial difficulties & 0,05 & 0,58 & 0,13 & $-0,23$ & 0,14 \\
\hline Work-family conflict because of study/work & 0,12 & $-0,01$ & 0,34 & 0,25 & $-0,11$ \\
\hline Enjoy studying & 0,00 & 0,03 & 0,16 & $-0,10$ & 0,67 \\
\hline Collegial and open atmosphere in the environment & $-0,10$ & 0,12 & 0,01 & 0,16 & 0,76 \\
\hline Support from family/partner in professional matters & $-0,14$ & 0,04 & $-0,03$ & 0,03 & 0,69 \\
\hline Cronbach's $\alpha$ & .84 & .75 & .70 & .40 & .72 \\
\hline \multicolumn{6}{|c|}{$\begin{array}{l}\text { Note. Extraction method = principal axis factors. Rotation method = oblique promax. Factor loadings }>.40 \text { are in bold. } \\
\text { Five factors explained a total of } 83 \% \text { of the variance. } N=183 \text {. Bold marking means that the stressors elicited the } \\
\text { strongest response from the data. Factor A physical strain, Factor B psycho-social strain, Factor C conflicts, } \\
\text { Factor D organizational strain, and Factor E resources. }\end{array}$} \\
\hline
\end{tabular}


Table $1 b$

Frequency of stress factors in the total sample and the subgroups of students of German and international students

\begin{tabular}{|c|c|c|c|c|}
\hline \multirow{2}{*}{$\begin{array}{c}\text { Presence } \\
\text { of stress factor }\end{array}$} & Total & Germany & Freign Countries & \multirow{2}{*}{ PMann-Whitney-U } \\
\hline \multirow{2}{*}{\multicolumn{5}{|c|}{$\begin{array}{l}\text { Frequency [\%] } \\
\text { Time pressure }\end{array}$}} \\
\hline & & & & \\
\hline never & 0,5 & 0,9 & 0 & \multirow[t]{4}{*}{0,524} \\
\hline rare & 24,2 & 27,2 & 20,0 & \\
\hline sometimes & 41,2 & 40,4 & 42,5 & \\
\hline often & 34,0 & 31,6 & 37,5 & \\
\hline \multicolumn{5}{|c|}{ Dirt pollution } \\
\hline never & 57,2 & 50,0 & 67,5 & \multirow[t]{4}{*}{0,078} \\
\hline rare & 25,8 & 30,7 & 18,8 & \\
\hline sometimes & 13,9 & 14,9 & 12,5 & \\
\hline often & 3,1 & 4,4 & 1,3 & \\
\hline \multicolumn{5}{|c|}{ Physically heavy work } \\
\hline never & 41,2 & 32,5 & 53,8 & \multirow[t]{4}{*}{0,007} \\
\hline rare & 31,4 & 39,5 & 20,0 & \\
\hline sometimes & 22,7 & 24,6 & 20,0 & \\
\hline often & 4,6 & 3,5 & 6,3 & \\
\hline \multicolumn{5}{|c|}{ Lifting/carrying } \\
\hline never & 45,4 & 34,2 & 61,3 & \multirow[t]{4}{*}{0,001} \\
\hline rare & 30,4 & 39,5 & 17,5 & \\
\hline sometimes & 18,0 & 21,1 & 13,8 & \\
\hline often & 6,2 & 5,3 & 7,5 & \\
\hline \multicolumn{5}{|c|}{ Unfavourable working hours } \\
\hline never & 29,4 & 30,7 & 27,5 & \multirow[t]{4}{*}{0,299} \\
\hline rare & 21,1 & 24,6 & 16,3 & \\
\hline sometimes & 36,1 & 20,1 & 16,0 & \\
\hline often & 13,4 & 10,5 & 17,5 & \\
\hline \multicolumn{5}{|c|}{ Incompatibility of tasks } \\
\hline never & 23,7 & 21,9 & 26,3 & 0,001 \\
\hline rare & 30,4 & 38,6 & 18,8 & \\
\hline sometimes & 34,5 & 34,2 & 35,0 & \\
\hline often & 11,3 & 5,3 & 20,0 & \\
\hline & & substance & & \\
\hline never & 72,2 & 75,4 & 67,5 & 0,683 \\
\hline rare & 21,6 & 19,3 & 25,0 & \\
\hline sometimes & 4,1 & 3,5 & 5,0 & \\
\hline often & 2,1 & 1,8 & 2,5 & \\
\hline & & till for long & & \\
\hline never & 13,4 & 4,4 & 26,3 & $<0,001$ \\
\hline rare & 19,1 & 15,8 & 23,8 & \\
\hline sometimes & 28,4 & 23,7 & 35,0 & \\
\hline often & 39,2 & 56,1 & 15,0 & \\
\hline & & nt risks exp & & \\
\hline never & 53,6 & 40,4 & 72,5 & $<0,001$ \\
\hline rare & 27,8 & 34,2 & 18,8 & \\
\hline sometimes & 13,9 & 18,4 & 7,5 & \\
\hline often & 4,6 & 7,0 & 1,3 & \\
\hline & & ess, hotnes & etc.) & \\
\hline never & 25,8 & 28,9 & 21,3 & $<0,001$ \\
\hline rare & 28,4 & 39,5 & 12,5 & \\
\hline sometimes & 25,8 & 22,8 & 30,0 & \\
\hline often & 20,1 & 8,8 & 36,3 & \\
\hline & & Monotony & & \\
\hline never & 25,8 & 28,1 & 22,5 & 0,487 \\
\hline rare & 36,6 & 36,8 & 36,3 & \\
\hline sometimes & 28,4 & 24,6 & 33,8 & \\
\hline often & 9,3 & 10,5 & 7,5 & \\
\hline & & orkoverloac & & \\
\hline never & 11,9 & 10,5 & 13,8 & $<0,001$ \\
\hline rare & 34,5 & 46,5 & 17,5 & \\
\hline sometimes & 43,3 & 38,6 & 50,0 & \\
\hline often & 10,3 & 4,4 & 18,8 & \\
\hline & & of informa & & \\
\hline never & 21,1 & 19,3 & 23,8 & 0,383 \\
\hline rare & 30,4 & 35,1 & 23,8 & \\
\hline sometimes & 37,1 & 34,2 & 41,3 & \\
\hline often & 11,3 & 11,4 & 11,3 & \\
\hline
\end{tabular}


continuation Table $1 b$

\begin{tabular}{|c|c|c|c|c|}
\hline \multicolumn{5}{|c|}{ Conflicts with faculty } \\
\hline never & 65,8 & 62,7 & 70,0 & \multirow[t]{4}{*}{0,018} \\
\hline rare & 27,4 & 33,6 & 18,8 & \\
\hline sometimes & 6,8 & 3,6 & 11,3 & \\
\hline often & 0 & 0 & 0 & \\
\hline \multicolumn{5}{|c|}{ Conflict with colleagues } \\
\hline never & 67,5 & 60,4 & 77,5 & \multirow[t]{4}{*}{0,037} \\
\hline rare & 25,1 & 32,4 & 15,0 & \\
\hline sometimes & 6,8 & 6,3 & 7,5 & \\
\hline often & 0,5 & 0,9 & 0 & \\
\hline \multicolumn{5}{|c|}{ Conflict with customers/patients } \\
\hline never & 81,1 & 73,6 & 91,3 & \multirow[t]{4}{*}{0,021} \\
\hline rare & 12,1 & 17,3 & 5,0 & \\
\hline sometimes & 6,3 & 8,2 & 3,8 & \\
\hline often & 0,5 & 0,9 & 0 & \\
\hline \multicolumn{5}{|c|}{ Social isolation } \\
\hline never & 33,7 & 47,8 & 13,8 & \multirow[t]{4}{*}{$<0,001$} \\
\hline rare & 20,7 & 29,2 & 8,8 & \\
\hline sometimes & 26,9 & 18,6 & 38,8 & \\
\hline often & 18,7 & 4,4 & 38,8 & \\
\hline \multicolumn{5}{|c|}{ Emotional stress } \\
\hline never & 14,5 & 18,6 & 8,8 & \multirow[t]{4}{*}{$<0,001$} \\
\hline rare & 32,6 & 45,1 & 15,0 & \\
\hline sometimes & 30,1 & 27,4 & 33,8 & \\
\hline often & 22,8 & 8,8 & 42,5 & \\
\hline \multicolumn{5}{|c|}{ Financial difficulties } \\
\hline never & 27,5 & 25,7 & 30,0 & \multirow[t]{4}{*}{0,012} \\
\hline rare & 24,4 & 31,9 & 13,8 & \\
\hline sometimes & 28,5 & 28,3 & 28,7 & \\
\hline often & 19,7 & 14,2 & 27,5 & \\
\hline \multicolumn{5}{|c|}{ Enjoy studying } \\
\hline never & 1,6 & 0,9 & 2,5 & \multirow[t]{4}{*}{$<0,001$} \\
\hline rare & 10,9 & 4,5 & 20,0 & \\
\hline sometimes & 41,1 & 35,7 & 48,8 & \\
\hline often & 46,4 & 58,9 & 28,7 & \\
\hline \multicolumn{5}{|c|}{ Collegial and open atmosphere in the environment } \\
\hline never & 2,1 & 0 & 5,0 & \multirow[t]{4}{*}{$<0,001$} \\
\hline rare & 9,4 & 6,3 & 13,8 & \\
\hline sometimes & 37,0 & 31,3 & 45,0 & \\
\hline often & 51,6 & 62,5 & 36,3 & \\
\hline \multicolumn{5}{|c|}{ Support from family/partner in professional matters } \\
\hline never & 4,7 & 3,5 & 6,3 & \multirow[t]{4}{*}{$<0,001$} \\
\hline rare & 9,3 & 6,2 & 13,8 & \\
\hline sometimes & 28,5 & 21,2 & 38,8 & \\
\hline often & 57,5 & 69,0 & 41,3 & \\
\hline
\end{tabular}

and accident risk exposure. Resources were also more available for German students than for international students. The international students were more strained (sometimes and often) by social isolation emotional stress and incompatibility of tasks than the Germans.

After recording the stressors, the intensity of the demands resulting from them was asked. The stressors that elicited the strongest responses were social isolation (25.9\% "very strong" and $20.7 \%$ "quite strong"), emotional stress (26.6\% "very strong" and "quite strong") and financial difficulties (24.0\% "very strong" and $23.4 \%$ "quite strong"). The perceived intensity of the stressors varied greatly in the groups of different origins.
Here too, a factor analysis was performed for the items measuring intensity of stressors. The KMO was 0.793 , and Bartlett's test was significant at $p<.001$. A factor analysis with $P F$ method was performed on the 34 variables measuring intensity of strains. Parallel analysis indicated that five factors should be extracted. Together they explained $84 \%$ of the variance in the data. A promax rotation gave 25 variables with factor loadings higher than 0.4 , without cross-loadings. The factors and their related items are presented in Table $2 a$. All factor had Cronbach's $\alpha$ above .70, apart from Factor D, with $\alpha=.55$. The Table $2 b$ shows the expression of stress caused by the different stress factors in 
Table $2 a$

Factor analysis of items measuring intensity of stress factors among students

\begin{tabular}{|c|c|c|c|c|c|}
\hline Variable & Factor A & Factor B & Factor C & Factor D & Factor $\mathrm{E}$ \\
\hline Time pressure & 0,10 & 0,74 & 0,00 & $-0,20$ & 0,06 \\
\hline Noise exposure & 0,43 & 0,05 & 0,04 & 0,03 & 0,02 \\
\hline Dirt pollution & 0,76 & $-0,02$ & $-0,04$ & $-0,06$ & $-0,05$ \\
\hline Physically heavy work & 0,82 & 0,08 & $-0,04$ & $-0,15$ & 0,03 \\
\hline Forced posture & 0,44 & 0,11 & $-0,11$ & 0,25 & 0,07 \\
\hline Lifting/carrying & 0,81 & 0,01 & 0,03 & $-0,21$ & $-0,16$ \\
\hline Unfavourable working hours & 0,27 & 0,50 & $-0,16$ & 0,13 & $-0,06$ \\
\hline Incompatibility of tasks & 0,16 & 0,47 & 0,08 & 0,11 & 0,00 \\
\hline Dangerous substances pollution & 0,50 & $-0,04$ & 0,21 & 0,17 & $-0,05$ \\
\hline Sitting still for long periods & 0,04 & $-0,12$ & $-0,18$ & 0,37 & 0,21 \\
\hline Long daily journey & 0,21 & 0,00 & 0,08 & 0,15 & 0,14 \\
\hline Risk for infection & 0,18 & $-0,10$ & 0,05 & 0,20 & 0,00 \\
\hline Accident risks exposure & 0,48 & $-0,25$ & 0,13 & 0,18 & 0,02 \\
\hline Climate (coldness, hotness, draft, etc.) & 0,05 & 0,53 & $-0,23$ & 0,24 & $-0,09$ \\
\hline Monotony & 0,10 & 0,26 & $-0,02$ & 0,30 & $-0,02$ \\
\hline Work overload & 0,08 & 0,59 & $-0,05$ & 0,12 & 0,07 \\
\hline Work underload & $-0,13$ & $-0,13$ & 0,14 & 0,58 & $-0,24$ \\
\hline High accountability & 0,11 & 0,60 & $-0,02$ & $-0,14$ & 0,14 \\
\hline $\begin{array}{l}\text { Little room for maneuver, no organization } \\
\text { possibilities }\end{array}$ & $-0,10$ & 0,34 & $-0,11$ & 0,44 & 0,15 \\
\hline Lack of information & $-0,14$ & 0,06 & $-0,11$ & 0,58 & 0,22 \\
\hline Sexual harassment & $-0,11$ & $-0,06$ & 0,20 & 0,49 & $-0,16$ \\
\hline Conflicts with faculty & 0,00 & $-0,08$ & 0,65 & 0,08 & 0,10 \\
\hline Conflicts with colleagues & $-0,03$ & 0,04 & 0,80 & 0,02 & $-0,05$ \\
\hline Conflicts with customers/patients & 0,05 & 0,05 & 0,65 & $-0,27$ & 0,07 \\
\hline Social isolation & $-0,22$ & 0,76 & 0,07 & 0,00 & $-0,06$ \\
\hline Fear of unemployment & 0,04 & 0,23 & 0,23 & 0,24 & $-0,07$ \\
\hline Emotional stress & $-0,09$ & 0,78 & 0,17 & $-0,03$ & $-0,09$ \\
\hline Problems in dealing with personal computers & $-0,11$ & 0,14 & 0,12 & 0,29 & 0,04 \\
\hline Bullying & 0,01 & 0,23 & 0,35 & 0,13 & 0,07 \\
\hline Financial difficulties & $-0,03$ & 0,36 & 0,17 & 0,06 & 0,13 \\
\hline Work-family conflict because of study/work & 0,09 & $-0,04$ & 0,22 & 0,14 & 0,29 \\
\hline Enjoy studying & 0,11 & $-0,05$ & 0,03 & $-0,11$ & 0,74 \\
\hline Collegial and open atmosphere in the environment & $-0,10$ & $-0,03$ & 0,01 & 0,04 & 0,82 \\
\hline Support from family/partner in professional matters & $-0,14$ & 0,08 & 0,01 & 0,01 & 0,75 \\
\hline Cronbach's a & .78 & .85 & .76 & .55 & .82 \\
\hline \multicolumn{6}{|c|}{$\begin{array}{l}\text { Note. Extraction method = principal axis factors. Rotation method = oblique promax. Factor loadings }>.40 \text { are in bold. } \\
\text { Five factors explaind a total of } 84 \% \text { of the variance. } N=188 \text {. Bold marking means that the stressors elicited the } \\
\text { strongest response from the data. Factor A physical strain, Factor B psycho-social strain, Factor } C \text { conflicts, Factor D } \\
\text { organizational strain, and Factor E resources. }\end{array}$} \\
\hline
\end{tabular}

comparison of the German and international students. Highly significant differences were found in the stress factors time pressure, unfavourable working hours, climate, work overload, high accountability, social isolation, and emotional stress. Here, the international students gave higher values than the German students. General conditions had an additional influence in the stress factors physically heavy work, lifting/ carrying, conflicts with customer/patients.

There was a large degree of overlap between the contents of the factors from the two analyses. Factor A and B shared most items, though the analyses showed one or two items loaded uniquely high on either frequency or intensity. Factor $\mathrm{C}$ and E were identical. Factor D, however, had only one item in common among the two factor analyses. It was also the factor with the worst internal reliability. Since the reliability scores of Factor D: organi- zational strain were so low $(\alpha=.55)$, they were excluded from further analysis.

We made sum scores of the items with loadings greater than .40 on each of the factors. Then we tested regression models where the factor scores were regressed on the students' origins and general conditions. Here too, in order to answer the question of what influence the generally stressful conditions have on the experience of stressors in the study, the variable general condition was included in the GLM analysis as a covariate. The results of the frequency factors are shown in Table 3, and the results of the intensity factors are shown in Table 4.

We ran regression models with origin predicting Factor A-E stressors, with robust standard errors in order to deal with heteroskedasticity and non-normality of residuals that were present in the data. In these models, we 
Table $2 b$

Expression of stress caused by the different stress factors in the total sample and in the group comparison of students from different backgrounds and with different framework conditions for study

\begin{tabular}{|c|c|c|c|c|c|c|c|}
\hline \multirow{2}{*}{ Beansprucht durch... } & Total & Germany & $\begin{array}{l}\text { Foreign } \\
\text { countries }\end{array}$ & \multirow{2}{*}{$\begin{array}{c}\text { PMann- } \\
\text { Whitney-U }\end{array}$} & \multicolumn{2}{|c|}{$\begin{array}{c}\text { GLM with general } \\
\text { conditions as covariate }\end{array}$} & \multirow{3}{*}{$\begin{array}{l}p_{\text {Bonferroni }} \\
<0.001\end{array}$} \\
\hline & \multicolumn{3}{|c|}{$\begin{array}{c}\mathrm{AV} \pm \mathrm{SD} \\
\text { Median (min-max) }\end{array}$} & & $\mathrm{F}$ & $\mathrm{p}$ & \\
\hline Time pressure & \begin{tabular}{|c|}
$1.57 \pm 0.856$ \\
$1(1-2)$ \\
\end{tabular} & $\begin{array}{l}1.24 \pm 0.747 \\
1(0-3)\end{array}$ & $\begin{array}{l}2.05 \pm 0.794 \\
2(0-3) \\
\end{array}$ & $<0.001$ & 24.237 & $<0.001$ & \\
\hline Noise exposure & $\begin{array}{c}0.69 \pm 0.863 \\
0(0-3) \\
\end{array}$ & $\begin{array}{c}0.60 \pm 0.822 \\
0(0-3)\end{array}$ & $\begin{array}{l}0.78 \pm 0.927 \\
0.5(0-3) \\
\end{array}$ & 0.367 & 0.940 & 0.393 & 0.175 \\
\hline Dirt pollution & $\begin{array}{c}0.28 \pm 0580 \\
0(0-3)\end{array}$ & $\begin{array}{c}0.22 \pm 0.488 \\
0(0-2)\end{array}$ & $\begin{array}{l}0.35 \pm 0.695 \\
0(0-3)\end{array}$ & 0.409 & 3.921 & 0.022 & 0.071 \\
\hline Physically heavy work & $\begin{array}{c}0.48 \pm 0.743 \\
0(0-3) \\
\end{array}$ & $\begin{array}{c}0.36 \pm 0.542 \\
0(0-2)\end{array}$ & $\begin{array}{l}0.69 \pm 0.936 \\
0(0-3)\end{array}$ & 0.031 & 14.529 & $<0.001$ & $<0.001$ \\
\hline Forced posture & $\begin{array}{c}0.79 \pm 0.934 \\
0(0-3)\end{array}$ & $\begin{array}{c}0.69 \pm 0.866 \\
0(0-3)\end{array}$ & $\begin{array}{l}0.89 \pm 0.968 \\
1(0-3) \\
\end{array}$ & 0.220 & 4.823 & 0.009 & 0.062 \\
\hline Lifting/Carrying & $\begin{array}{c}0.48 \pm 0.707 \\
0(0-3)\end{array}$ & $\begin{array}{c}0.45 \pm 0.594 \\
0(0-2)\end{array}$ & $\begin{array}{l}0.53 \pm 0.842 \\
0(0-3)\end{array}$ & 0.848 & 12.063 & $<0.001$ & 0.122 \\
\hline $\begin{array}{l}\text { Unfavourable working } \\
\text { hours }\end{array}$ & $\begin{array}{c}1.04 \pm 0.986 \\
1(0-3)\end{array}$ & $\begin{array}{c}0.83 \pm 0.813 \\
1(0-3)\end{array}$ & $\begin{array}{l}1.4 \pm 1.121 \\
1.5(0-3)\end{array}$ & $<0.001$ & 12.079 & $<0.001$ & $<0.001$ \\
\hline Incompatibility of task & $\begin{array}{c}1.19 \pm 1.006 \\
1(0-3)\end{array}$ & $\begin{array}{c}1.12 \pm 0.944 \\
1(0-3)\end{array}$ & $\begin{array}{l}1.34 \pm 1.102 \\
1.5(0-3)\end{array}$ & 0.103 & 3.541 & 0.031 & 0.077 \\
\hline $\begin{array}{l}\text { Dangerous stubstances } \\
\text { pollution }\end{array}$ & $\begin{array}{c}0.25 \pm 0.539 \\
0(0-3)\end{array}$ & $\begin{array}{c}0.18 \pm 0.462 \\
0(0-2)\end{array}$ & $\begin{array}{l}0.36 \pm 0.641 \\
0(0-3)\end{array}$ & 0.012 & 4.253 & 0.016 & 0.014 \\
\hline Accident risk exposure & $\begin{array}{c}0.34 \pm 0.633 \\
0(0-3)\end{array}$ & $\begin{array}{c}0.39 \pm 0.603 \\
0(0-2)\end{array}$ & $\begin{array}{l}0.30 \pm 0.701 \\
0(0-3)\end{array}$ & 0.117 & 2.773 & 0.065 & 0.597 \\
\hline $\begin{array}{l}\text { Climate (coldness. } \\
\text { hotness. draft. etc.) }\end{array}$ & $\begin{array}{c}1.09 \pm 1.109 \\
1(0-3)\end{array}$ & $\begin{array}{c}0.71 \pm 0.786 \\
1(0-3) \\
\end{array}$ & $\begin{array}{l}1.66 \pm 1.252 \\
2(0-3)\end{array}$ & $<0.001$ & 19.442 & $<0.001$ & $<0.001$ \\
\hline Work overload & $\begin{array}{c}1.42 \pm 0.969 \\
(1(0-3)\end{array}$ & $\begin{array}{c}1.21 \pm 0.853 \\
1(0-3)\end{array}$ & $\begin{array}{l}1.71 \pm 1.058 \\
2(0-3)\end{array}$ & $<0.001$ & 6.339 & 0.002 & 0.001 \\
\hline Work underload & $\begin{array}{c}0.34 \pm 0.657 \\
0(0-3)\end{array}$ & $\begin{array}{c}0.40 \pm 0.654 \\
0(0-2)\end{array}$ & $\begin{array}{l}0.24 \pm 0.661 \\
0(0-3)\end{array}$ & 0.009 & 2.832 & 0.062 & 0.190 \\
\hline High accountability & $\begin{array}{c}0.97 \pm 0.921 \\
1(0-3) \\
\end{array}$ & $\begin{array}{c}0.60 \pm 0.670 \\
1(0-3) \\
\end{array}$ & $\begin{array}{l}1.51 \pm 0.968 \\
2(0-3) \\
\end{array}$ & $<0.001$ & 27.730 & $<0.001$ & $<0.001$ \\
\hline $\begin{array}{l}\text { Litte room for maneuver. } \\
\text { no organization } \\
\text { possibilities }\end{array}$ & $\begin{array}{c}0.98 \pm 1.018 \\
1(0-3)\end{array}$ & $\begin{array}{c}0.83 \pm 0.953 \\
1(0-3)\end{array}$ & $\begin{array}{c}1.21 \pm 1.076 \\
1(0-3)\end{array}$ & 0.010 & 3.254 & 0.041 & 0.016 \\
\hline Lack of informtion & \begin{tabular}{|c|}
$1.42 \pm 1.013$ \\
$2(0-3)$ \\
\end{tabular} & $\begin{array}{c}1.45 \pm 0.975 \\
2(0-3)\end{array}$ & $\begin{array}{c}1.41 \pm 1.064 \\
2(0-3) \\
\end{array}$ & 0.929 & 0.048 & 0.954 & 0.790 \\
\hline Sexual harassment & $\begin{array}{c}0.10 \pm 0.414 \\
0(0-3)\end{array}$ & $\begin{array}{c}0.12 \pm 0.460 \\
0(0-3)\end{array}$ & $\begin{array}{c}0.09 \pm 0.396 \\
0(0-3)\end{array}$ & 0.822 & 1.785 & 0.171 & 0.820 \\
\hline Conflicts with faculty & $\begin{array}{c}0.51 \pm 0.888 \\
0(0-3)\end{array}$ & $\begin{array}{c}0.50 \pm 0.815 \\
0(0-3)\end{array}$ & $\begin{array}{c}0.54 \pm 0.993 \\
0(0-3)\end{array}$ & 0.709 & 4.610 & 0.011 & 0.433 \\
\hline Conflicts with colleagues & $\begin{array}{c}0.40 \pm 0.760 \\
0(0-3)\end{array}$ & $\begin{array}{c}0.43 \pm 0.746 \\
0(0-3)\end{array}$ & $\begin{array}{c}0.35 \pm 0.797 \\
0(0-3) \\
\end{array}$ & 0.152 & 2.061 & 0.130 & 0.725 \\
\hline $\begin{array}{l}\text { Conflicts with } \\
\text { customer/patients }\end{array}$ & $\begin{array}{c}0.23 \pm \\
0.616 \\
0(0-3) \\
\end{array}$ & $\begin{array}{c}0.32 \pm 0.683 \\
0(0-3)\end{array}$ & $\begin{array}{c}0.14 \pm 0.522 \\
0(0-3)\end{array}$ & 0.021 & 9.004 & $<0.001$ & 0.182 \\
\hline Social isolation & $\begin{array}{c}1.33 \pm \\
1.243 \\
1(0-3) \\
\end{array}$ & $\begin{array}{c}0.82 \pm 1.049 \\
0(0-3)\end{array}$ & $\begin{array}{c}2.09 \pm 1.127 \\
3(0-3)\end{array}$ & $<0.001$ & 32.006 & $<0.001$ & $<0.001$ \\
\hline Emotional stress & $\begin{array}{c}1.59 \pm \\
1.094 \\
2(0-3) \\
\end{array}$ & $\begin{array}{c}1.27 \pm 1.021 \\
1(0-3)\end{array}$ & $\begin{array}{c}2.09 \pm 0.983 \\
2(0-3)\end{array}$ & $<0.001$ & 14.702 & $<0.001$ & $<0.001$ \\
\hline Enjoy studying & $\begin{array}{c}0.53 \pm 0.876 \\
0(0-3) \\
\end{array}$ & $\begin{array}{c}0,41 \pm 0.752 \\
0(0-3) \\
\end{array}$ & $\begin{array}{c}0.69 \pm 1.001 \\
0(0-3)\end{array}$ & 0.080 & 2.654 & 0.051 & 0.032 \\
\hline $\begin{array}{l}\text { Collegial and open } \\
\text { atmosphere in the } \\
\text { environment }\end{array}$ & $\begin{array}{c}0.39 \pm 0.770 \\
0(0-3)\end{array}$ & $\begin{array}{c}0.29 \pm 0.644 \\
0(0-3)\end{array}$ & $\begin{array}{l}0.51 \pm 0.900 \\
0(0-3)\end{array}$ & 0.081 & 0.909 & 0.438 & 0.050 \\
\hline $\begin{array}{l}\text { Support from } \\
\text { family/partner in } \\
\text { professional matters }\end{array}$ & $\begin{array}{c}0.38 \pm 0.761 \\
0(0-3) \\
\end{array}$ & $\begin{array}{c}0.27 \pm 0.574 \\
0(0-3)\end{array}$ & $\begin{array}{l}0.53 \pm 0.941 \\
0(0-3)\end{array}$ & 0.128 & 0.581 & 0.619 & 0.022 \\
\hline
\end{tabular}


Table 3

Frequency of different stress factors in the overall sample and the subgroups of students of German and foreign origin

\begin{tabular}{|c|c|c|c|c|c|c|c|c|c|c|}
\hline \multirow{5}{*}{$\begin{array}{l}\text { Factor A: } \\
\text { Physical strain } \\
\text { Factor B: } \\
\text { Psycho-social } \\
\text { strain }\end{array}$} & Total & Germany & $\begin{array}{l}\text { Foreign } \\
\text { countries }\end{array}$ & \multicolumn{2}{|c|}{ Mann-Whitney-U } & \multicolumn{5}{|c|}{ GLM with general conditions as covariate } \\
\hline & \multicolumn{3}{|c|}{$\begin{array}{c}\mathrm{AV} \pm \mathrm{SD} \\
\text { Median (min-max) }\end{array}$} & $z$ & $p$ & $F$ & $R^{2}$ & $p$ & $b_{\circ}$ & $b_{\mathrm{GC}}$ \\
\hline & $\begin{array}{l}0.69 \pm 0.67 \\
0.6(0-2.8)\end{array}$ & $\begin{array}{c}0.79 \pm 0.65 \\
0.6(0-2.8)\end{array}$ & $\begin{array}{c}0.55 \pm 0.67 \\
0.2(0-2.8)\end{array}$ & 3.14 & .002 & 31.89 & .26 & $<.001$ & -.15 & $.64^{\star \star \star}$ \\
\hline & $1.50 \pm 0.60$ & $1.3 \pm 0.45$ & $1.79 \pm 0.66$ & -5.21 & $<.001$ & 27.71 & .23 & $<.001$ & $.53^{* * *}$ & $.30^{* * *}$ \\
\hline & $1.38(0.13-3)$ & $1.31(0.13-2.25)$ & $1.88(0.38-3)$ & & & & & & & \\
\hline $\begin{array}{l}\text { Factor C: } \\
\text { Conflicts }\end{array}$ & $\begin{array}{c}0.36 \pm 0.49 \\
0(0-2)\end{array}$ & $\begin{array}{c}0.42 \pm 0.5 \\
0.33(0-2)\end{array}$ & $\begin{array}{c}0.28 \pm 0.47 \\
0(0-2)\end{array}$ & 2.21 & .026 & 11.93 & .11 & $<.001$ & -.10 & $.30^{* * *}$ \\
\hline $\begin{array}{l}\text { Factor E: } \\
\text { Resources }\end{array}$ & $\begin{array}{c}2.36 \pm 0.62 \\
2.5(0-3)\end{array}$ & $\begin{array}{l}2.55 \pm 0.46 \\
2.67(1-3)\end{array}$ & $\begin{array}{c}2.1 \pm 0.72 \\
2(0-3)\end{array}$ & 4.49 & $<.001$ & 12.01 & .13 & $<.001$ & $-.43^{* * *}$ & .06 \\
\hline
\end{tabular}

German students and students without general conditions, respectively. $(N=191-194)$.

Table 4

Intensity of stress factors in the overall sample and the subgroups of students of German and foreign origin

\begin{tabular}{|c|c|c|c|c|c|c|c|c|c|c|}
\hline \multirow[b]{3}{*}{$\begin{array}{l}\text { Factor A: } \\
\text { Physical strain }\end{array}$} & Total & Cormani & \multirow{2}{*}{$\begin{array}{c}\text { Foreign } \\
\text { countries }\end{array}$} & \multicolumn{2}{|c|}{ Mann-Whitney-U } & \multicolumn{5}{|c|}{ GLM with general conditions as covariate } \\
\hline & & $\begin{array}{c}\mathrm{AV} \pm \mathrm{SD} \\
\mathrm{dian}(\mathrm{min}-\mathrm{max})\end{array}$ & & $z$ & $p$ & $F$ & $R^{2}$ & $p$ & $b_{\circ}$ & $b_{\mathrm{GC}}$ \\
\hline & $\begin{array}{c}0.47 \pm 0.48 \\
0.29(0-2.57)\end{array}$ & $\begin{array}{c}0.41 \pm 0.36 \\
0.29(0-1.43)\end{array}$ & $\begin{array}{c}0.56 \pm 0.6 \\
0.29(0-2.57)\end{array}$ & $-0,71$ & ,480 & 10,29 & ,11 & $<.001$ & $.18^{*}$ & $.28^{* * *}$ \\
\hline $\begin{array}{l}\text { Factor B: } \\
\text { Psychosocial }\end{array}$ & $1.27 \pm 0.71$ & $0.95 \pm 0.49$ & $1.73 \pm 0.73$ & $-7,14$ & $<.001$ & 34,44 & ,30 & $<.001$ & $.79^{\star * *}$ &, 11 \\
\hline strain & $1.19(0-3)$ & $0.94(0-2.38)$ & $1.88(0.25-3)$ & & & & & & & \\
\hline $\begin{array}{l}\text { Factor C: } \\
\text { Conflicts }\end{array}$ & $\begin{array}{c}0.38 \pm 0.62 \\
0(0-3)\end{array}$ & $\begin{array}{c}0.41 \pm 0.63 \\
0(0-3)\end{array}$ & $\begin{array}{c}0.34 \pm 0.61 \\
0(0-2)\end{array}$ & 0,98 & ,327 & 5,52 & ,06 & $<.001$ &,- 03 & $.30^{* *}$ \\
\hline $\begin{array}{l}\text { Factor E: } \\
\text { Resources }\end{array}$ & $\begin{array}{c}0.43 \pm 0.69 \\
0(0-3)\end{array}$ & $\begin{array}{c}0.33 \pm 0.58 \\
0(0-2.5)\end{array}$ & $\begin{array}{c}0.58 \pm 0.81 \\
0(0-3)\end{array}$ & $-2,23$ & ,026 & 2,73 & ,03 & ,068 & $.25^{*}$ &, 01 \\
\hline
\end{tabular}

adjusted for the effect of generally stressful conditions and background (German or international). For frequency of stressors, international students experienced psychosocial strain more often than German students did $(b=0.53, p<.001)$. German students experienced resources $(b=-0.43, p<.001)$ more often than international students. These with one or more generally stressful conditions experienced physical strain $(b=0.64, p<.001)$, psychosocial strain $(b=0.30$, $\mathrm{p}<.001)$ and conflicts $(\mathrm{b}=0.30, \mathrm{p}<.001)$ more often than those without. For intensity of stressors, international students experienced physical strain slightly more than German students ( $b=018, p<.05$ ), and psychosocial strain much more strongly $(b=0.79, p<.001)$. However, they also experienced resources more strongly than German students did $(b=0.25, p<.05)$. These with generally stressful conditions experienced physical strain $(b=0.28$, $\mathrm{p}<.001)$ and conflicts $(\mathrm{b}=0.30, \mathrm{p}<.01)$ significantly more strongly.

\section{Conclusions}

The present study examined 194 students of German and international origin with regard to whether there were differences in study-related stress and demands. The study looked at whether additional generally stressful conditions, for example existing part time job, the presence of children or taking care of relatives and a daily travel time of 120 minutes or more to the university, increased these study-related burdens. Although only German students had children in comparison to the international students, we see only a small influence of this variable because it was only $2.1 \%$ of the total sample. Nearly $45 \%$ of the students reported at least one of the general conditions that were stressful. The results also showed that the main stress factors of the two groups of students differed significantly. In general, the international students experienced stress factors more often and more intensely than the German students did.

When looking at the items making up the factor of physical stress, there were differences in "physically heavy work", "lifting/carrying", "unfavorable working hours" and "incompatibility of tasks". This stress is more pronounced among international students than among their German fellow students. Considering the general conditions, this characteristic remains. We suspect that both German and international students have part-time work, but that the international students tends to have jobs that are physically more difficult or at least that they perceive to be physically more difficult. It is also possible that the working hours 
are unfavourable, with increased time pressure and possibly precarious working conditions. We hypothesized it; we found no study that examined this. Perhaps, the time pressure is exacerbated by the language barriers that international students must overcome to participate in class, answer questions, write term papers, essays, reports, dissertations, and theses. International students take longer to use the language of their host country, especially the academic language of their subject in the language of their host country, than do domestic students. Based on data from the German National Educational Panel Study, the language used at home does not affect the students' learning [21]. A study showed that young people are extra sensitive to health problems if they work in precarious conditions [22]. Precarious conditions can be study-related situations or due to a second job. Working on a job more than 20 to 25 hours a week increases the probability of dropping out of study and decreases the probability of timely graduation [23, 24].

They also reported more stress from time pressure, which was similar to values reported by 1 [1] and 25 [25]. The international students also more often reported emotional stress compared to the German students. Data used from seven waves of panel data from the German National Educational Panel Study (NEPS) with 6,386 individuals (included 1,002 international students) found no support for an immigrant-native gap in life satisfaction [26].

First, new role conflicts caused by breaking away from family and friends and the associated independence are suspected to be a crisis-prone biographical phase between adolescence and adulthood [11], which appears to be particularly significant for international students. Overcoming this causes orientation difficulties in the formation of one's own identity [11]. In addition, role conflicts also arise, where students try to perform several social roles at the same time. In this context, the contradictions between the effort of special personal initiatives and self-responsibility and the restrictive requirements of institutional rules and examination regulations have a particularly strong effect [27]. The fact, that many decisions that have a long-term impact on future professional life must be made before this development phase is completed, presents students with further challenges. Thus, fears and coping limits cross each other [11]. The high incidence of social isolation (e.g., due to insufficient integration) among nearly $78 \%$ of international students (vs. $23 \%$ German students) could also speak for this.
Reasons for problems with integration in their own country and culture at Germans students are for example: not studying at the university of choice, greater distances between home and place of study, more frequent journeys back to home, knowledge of returning home and thus avoidance of social contacts. There is also probably a high pressure of expectation or pressure not to disappoint, as the family pays for the financial costs of the study to a larger degree and thus and enables their child to study in a foreign country. Studies showed that the success of individual students is influenced by school and family characteristics [28], but the immigration background has only a minor effect on study success [29].

There has been an increase in performance problems after the Bologna reform, which is accompanied by a growing dissatisfaction with the decreasing autonomy in the choice for their study content, insufficient study conditions and a general condensation of the material of learning [10].

Almost $69 \%$ of international and $43 \%$ of the German students in our sample stated that they were overworked sometimes or often. The autonomy of the students is limited in the long term by the modularization of the study programs, which also leads to a lower expectation of selfefficacy [10]. In our sample, only $28 \%$ of German students, but almost half of the international students reported a lack of room for maneuver and organizational possibilities. Even if the general conditions are taken into account, group differences were found in the stressors of time pressure, climate, high accountability and social isolation. It is also possible that social integration factors play a central role in explaining the different characteristics of stress factors and their stress profile between students of different origins. A study of over 70,000 Swedes found important indicators of poorer mental health in social integration: low social activity, lack of trust in others, lack of social support or financial burden [30]. In a study of international medical students at German universities, the language barrier was their main challenge [31].

The limitations of the study are that it did not take into account the exact time of the semester at which the survey of individual students was undertaken. For example, surveys at the end of the semester can result in higher demands than at the beginning, as the general conditions have already been in place for several weeks and, especially at the end of the semester, examinations and oral exams may also be required. For international students, holiday at home could have 
a positive effect on the stress level, which is most likely to be lower at the beginning of the semester than at the end. Four factors were considered as generally stressful conditions, which were cited by the total sample: working alongside studying, caring for children, nursing relatives and travel distance to place of work or study of more than 120 minutes. It should be noted that the international students in this study had no children to care for during their full-time study in Germany. The methodology does not allow to provide explanations for their findings. The results are compared with other studies.

The results showed that international students are more exposed to physical stressors. The type of secondary employment was not queried. It is assumed that international students are more likely to have unfavourable and physically more burdensome secondary jobs (for example waiters, student assistant). The questionnaires were presented in paper format, which offer less options of filling it out anonymously compared to a digital form. The risk of socially desirable answers might thus be elevated. The questions were in German, which may have made them harder for the international students to understand. Furthermore, it was not recorded in which semester the students were, which could also play a role. Medical students in their third or ninth semesters of medical school showed the highest values for perceived stress [32]. Nevertheless, the results illustrate the need for preventive and health promoting programs for students in a university setting- as the promotion of health and social competence [33]. However, we cannot generalize from this small study. Thus, we cannot make recommendations for political decisions. For this, larger studies that also look at students at the beginning, during the study period and at the end should be conducted. Data were collected before ongoing Covid 19 pandemic. In general, the strains on students are all still valid; are in some cases increased by the pandemic.

\section{List of abbreviations}

NEPS National Educational Panel Study

PF principal (axis) factors extraction method

Declarations:

Ethics approval and consent to participate

The Otto von Guericke University in Magdeburg, Germany (register no. 65/08) gave positive ethical opinions. The study complied with the guidelines of Declaration of Helsinki. The consent of the study participants was given in writing.

\section{Consent for publication}

All authors give their consent to publication.

\section{Funding Sources}

There are no sources of financing.

\section{Competing Interests}

The authors declare that there is no conflict of interest.

Availability of data and material (data transparency)

The data can be requested from the authors.

Authors' contributions

IB is the first author of the publication and responsible for data collection. HK performed statistical method and controlled the English version as a native speaker. BT made additions to the first version of publication and translated into English. SD is also co-editor the manuscript. All authors have read and approved the manuscript

\section{Acknowledgements}

We would like to thank Jessica Linke, Shawki Bahmad and Claudia Schupp, who were particularly involved in the initial phase of the study.

\section{References}

1. Kirsch, A.-S., Laemmert, P., Tittlbach, S. (2017). Gesundheitliche Anforderungen und Ressourcen von Studierenden [Student health requirements and resources]. Pr?v Gesundheitsf [Prev Health], 12, 181-188. doi: 10.1007/s11553-017-0584-3 [German].

2. Statista. Number of students at universities in Germany from 2002/2003 to 2018/2019. 2019. Retrieved from: https://de.statista.com/statistik/daten/studie/221/umfrage/anzahl-derstudenten-andeutschen-hochschulen. (Accessed 22 Feb 2020).

3. Holm-Hadulla, R.M., Hofmann, F.-H., Sperth, M., Funke, J. (2009). Psychische Beschwerden und Storungen von Studierenden [Psychological complaints and disorders of students]. Psychotherapeut [Psychotherapist], 54, 346-356. doi: 10.1007/s00278-009-0693-3 [German].

4. Schafer, A. [Schafer, A.] Psychische Belastung im Studium: Daten, Fakten, Handlungsfelder - ein Uberblick [Psychological stress during studies: data, facts, fields of action - an overview]. Dusseldorf [Dusseldorf]; 2013. Retrieved from: https://is.gd/kA1HcG [German]. 
5. Faller G. Gesundheit im Studium: Bedingungen und Ressourcen [Health in Studies: Conditions and Resources]. In: Faller G, Schnabel EP, editors. Wege zur gesunden Hochschule: Ein Leitfaden fur die Praxis [Paths to a Healthy College: A Practical Guide]. 1st ed. Berlin: Sigma, 2016. P. 49-57. Retrieved from: https://www.fachportal-paedagogik.de/literatur/vollanzeige.html?FId=786042 [German].

6. Messing B. Das Studium: Vom Start zum Ziel. Leitfaden fur Studierende [The course: from start to finish. Guide for students]. 2nd ed. Berlin: Springer, 2012. Retrieved from: https://www.springer.com/ gp/book/9783540294405 [German].

7. Braun, M., Laging, M., Heidenreich, T., Ganz, T. (2014). Gesundheitsberichterstattung an der Hochschule Esslingen am Beispiel der Studie "Stresserleben und Stressverarbeitung unter Studierenden" [Health reporting at the Esslingen University of Applied Sciences using the example of the study "Experiencing stress and coping with stress among students"]. Gesundheitswesen [Healthcare], 76, A15. doi: 10.1055/s-0034-1386865 [German].

8. Poskowsky, J. (2016). Datenlage zu mehrfach belasteten Studierenden [Data situation on students exposed to multiple stresses]. Fachtagung "Mehrfach belastete Studierende" [Symposium "Students exposed to multiple stresses"]. Germany, Paderborn. Retrieved from: http://www.gesundheitsfoerderndehochschulen.de/Inhalte/A_Arbeitskreis /A6_Tagungsberichte/16_09_30_ Paderborn/ Poskowsky_300916.pdf [German].

9. Burkhardt, S., Kercher, J. Abbruchquoten ausl?ndischer Studierender. Bonn: DAAD, 2014. 10 p. Retrieved from: https://is.gd/GT9RFd [German].

10. Klug, C., Strack, M., Reich, G. (2013). Belastungen von Bachelor- und Diplom-Studierenden [Burdens on Bachelor and Diploma students]. Psychotherapeut [Psychotherapist], 58, 159-164. doi: 10.1007/s00278-012-0914-z [German].

11. Ackermann, E., Schumann, W. (2010). University life's not a bed of roses. The psychosocial situation of German students. Praev Gesundheitsf [Praev Health], 5, 231-237. doi: 10.1007/s11553-0100234-5 [German].

12. Moreira, T., Foxcroft, D.R. (2008). The effectiveness of brief personalized normative feedback in reducing alcohol-related problems amongst University students: protocol for a randomized controlled trial. BMC Public Health, 8, 113. doi: 10.1186/1471-2458-8-113.

13. Grutzmacher, J. [Grutzmacher, J.], Gusy, B., Lesener, T., Sudheimer, S., Willige, J. (2018). Gesundheit Studierender in Deutschland 2017. Ein Kooperationsprojekt zwischen dem Deutschen Zentrum fur Hochschul- und Wissenschaftsforschung, der Freien Universit?t Berlin und der Techniker Krankenkasse [Health Students in Germany 2017. A cooperation project between the German Center for University and Science Research, the Free University of Berlin and the Techniker Krankenkasse]. Retrieved from: https:/ /www.ewi-psy.fu-berlin.de/einrichtungen/arbeitsbereiche/ppg/bwb-2017/_inhaltselemente/faktenblaetter/ Gesamtbericht-Gesundheit-Studierender-in-Deutschland-2017.pdf [German].

14. Stock, C. Wie bedeutsam ist Gesundheit fur den Studienerfolg von Studierenden? [How important is health for student success?] Praev Gesundheitsf [Prev Health], 12, 230-233. doi: 10.1007/s11553017-0609-y [German].

15. Herbst, U., Voeth, M., Eidhoff, A., Muller, M. [Muller, M.], Stief, S. (2013). Studierendenstress in Deutschland - Eine empirische Untersuchung [Student Stress in Germany - An Empirical Study]. Berlin: AOK Bundesverband. Retrieved from: https://www.aok-bv.de/imperia/md/aokbv/presse/ pressemitteilungen/archiv/2016/08_projektbericht_stressstudie_druck.pdf [German].

16. Hofmann, F.-H., Sperth, M., Holm-Hadulla, R.M. (2017). Psychische Belastungen und Probleme Studierender [Psychological stress and problems of students]. Psychotherapeut [Psychotherapist], 62, 395-402. doi: 10.1007/s00278-017-0224-6 [German].

17. Schmitt L. Bestellt und nicht abgeholt. Soziale Ungleichheit und Habitus-Struktur-Konflikte im Studium [Ordered and not picked up. Social inequality and habitus structure conflicts in studies]. Wiesbaden: GWV Fachverlage GmbH; 2013. Retrieved from: https:/www.springer.com/de/book/9783531171883 [German].

18. Galan, F. [Galan, F.], Sanmartin, A. [Sanmartin, A.], Polo, J., Giner, L. (2011). Burnout risk in medical students in Spain using the Maslach Burnout Inventory-Student Survey. Int Arch Occup Environ Health, 84, 453-459. doi: 10.1007/s00420-011-0623-x.

19. Luczak, H., Rohmert, W. Belastungs-Beanspruchungs-Konzepte [Stress-strain concepts]. In: Luczak H, Volpert W (Hrsg). Handbuch Arbeitswissenschaft [Manual of ergonomics]. Stuttgart: SchafferPoeschel, 1997. [German]. 
20. Muth, T., Siegmann, S., Schwarze, S. (2011). Was belastet Studierende der Humanmedizin? [What burdens students of human medicine?] Arbeitsmed.Sozialmed.Umweltmed [Occupational medicine, social medicine, environmental medicine], 46, 162-163.

21. Strobel, B. (2016). Does family language matter? The role of foreign language use and family social capital in the educational achievement of immigrant students in Germany. Ethnic and Racial Studies, 39, 2641-2663. doi: 10.1080/01419870.2016.1145712.

22. Vancea, M., Utzet, M. (2017). How unemployment and precarious employment affect the health of young people: A scoping study on social determinants. Scand J Public Health, 45, 73-84. doi: 10.1177/ 1403494816679555.

23. Beerkens, M., Magi, E. [Magi, E.], Lill, L. (2011). University studies as a side job: causes and consequences of massive student employment in Estonia. Higher Education, 61, 679-692. doi: 10.1007/ s10734-010-9356-0.

24. Hovdhaugen, E. (2015). Working while studying: the impact of term-time employment on dropout rates. Journal of Education and Work, 28, 631-651. doi: 10.1080/13639080.2013.869311.

25. Muth, T., Siegmann, S., Schwarze, S. (2007). Lebenssituation und berufliche Belastungen von Studierenden der Medizin [Living situation and occupational stresses of students of medicine]. Praktische Arbeitsmedizin [Practical occupational medicine], 9, 593-594 [German].

26. Granderath, J.S., Martin, A., Froehlich, L. (2021). The Effect of Participation in Adult Education on Life Satisfaction of Immigrants and Natives: A Longitudinal Analysis. Journal of Happiness Studies. doi: 10.1007/s10902-020-00340-7.

27. Klaner, A. Stressbewaltigung im Studium. Mit 20 praktischen Ubungen zum erfolgreichen Stressabbau [Coping with stress in studies. With 20 practical exercises to successfully reduce stress]. Berlin: A. Spitz, 1998. Retrieved from: https://is.gd/S2RK2F [German].

28. Prakhov, I., Sergienko, D. (2020). Matching between students and universities: What are the sources of inequalities of access to higher education? Eur J Educ, 55, 261-274. doi: 10.1111/ejed.12389.

29. Behr, A., Giese, M., Teguim, K.H.D., Theune, K. (2020). Dropping out from Higher Education in Germany an Empirical Evaluation of Determinants for Bachelor Students. Open Education Studies, 2, 126-148. doi: 10.1515/edu-2020-0104.

30. Brydsten, A., Rostila, M., Dunlavy, A. (2019). Social integration and mental health - a decomposition approach to mental health inequalities between the foreign-born and native-born in Sweden. Int J Equity Health, 18, 48. doi: 10.1186/s12939-019-0950-1.

31. Huhn, D., Junne, F., Zipfel, S., Duelli, R., Resch, F., Herzog, W., Nikendei, C. (2015). International medical students - a survey of perceived challenges and established support services at medical faculties. GMS Z Med Ausbild, 32, Doc9. doi: 10.3205/zma000951.

32. Erschens, R., Herrmann-Werner, A., Keifenheim, K.E., Loda, T., Bugaj, T.J., Nikendei, C. ... Junne, F. (2018). Differential determination of perceived stress in medical students and high-school graduates due to private and training-related stressors. PLoS ONE, 13, e0191831. doi: 10.1371/ journal.pone.0191831.

33. Elias, M.J. (1995). Primary prevention as health and social competence promotion. Journal of Primary Prevention, 16, 5-24. doi: 10.1007/BF02407230.

Received: 07-Apr-2021

Accepted: 20-Jun-2021 